\title{
Severe Tropical Infections
}

\author{
Dhruva Chaudhry, Diksha Tyagi, and Sushmitha Jakka
}

A 26-year-old female patient presented with high-grade fever for 10 days followed by pedal edema and decreased urine output for 4 days. She developed shortness of breath for last 2 days which was associated with dry cough. There was no history of paroxysmal nocturnal dyspnea and orthopnea. History of epistaxis was present for 1 day. No history of travel to another place was present. On examination, she was conscious, well oriented. Her pulse rate was $124 / \mathrm{min}$, blood pressure was $90 / 60 \mathrm{mmHg}$ and $\mathrm{SpO}_{2}$ was $86 \%$ on room air. Eschar was present under the left breast. On auscultation, bilateral crepitations were present. Cardiovascular examination revealed no abnormality. Blood Investigations revealed hemoglobin- $9.0 \mathrm{mg} / \mathrm{dl}$, platelet count $-70,000 / \mathrm{mm}^{3}$, blood urea $-108 \mathrm{mg} / \mathrm{dl}$, serum creatinine- $1.6 \mathrm{mg} / \mathrm{dl}$, blood sugar-91 mg/dl, SGOT/PT_440/235. X-ray chest was suggestive of bilateral diffuse alveolar shadows. Arterial blood gas analysis revealed $\mathrm{pH}-7.38, \mathrm{pO}_{2}-58 \mathrm{mmHg}, \mathrm{pCO}_{2}-44 \mathrm{mmHg}, \mathrm{HCO}_{3}-15 \mathrm{mmol} / \mathrm{L}$, $\mathrm{SaO}_{2}-86 \%$.

Severe tropical infections with multiorgan involvement, though common cause of admission in ICU but establishing etiology can be a challenge. Dengue, scrub typhus, encephalitis/meningitis, malaria etc. are the major tropical fevers in Indian ICUs. Close monitoring and supportive therapy with antibiotics where necessary, are the mainstay of treatment in most of these infections. Rapid identification of treatable infection is imperative for a better outcome.

D. Chaudhry $(\varangle) \cdot$ D. Tyagi $\cdot$ S. Jakka

Department of Pulmonary and Critical Care Medicine, Pt. B. D. Sharma Post Graduate Institute of Medical Sciences, Rohtak, Haryana, India 


\section{Step 1: Initial Assessment and Resuscitation}

- Assess for the airway, breathing and circulation.

- Fluid resuscitation is the mainstay of initial management in most of the tropical infections as they present late, may have predominant diarrheal component, and are usually dehydrated.

- Capillary leak syndrome is a known complication in many tropical infections.

- At the same time, close monitoring for volume overload and pulmonary edema should also be done.

- Patients presenting with encephalopathy syndromes need closer airway assessment and assisted ventilation.

- While resuscitation is going on, send investigations:

- Complete blood count-neutropenia and thrombocytopenia are common features in many tropical infections. Leptospirosis, scrub typhus usually have leukocytosis.

- C-reactive protein (CRP).

- Peripheral blood smear for Malarial parasite, dual malarial antigen.

- Dengue antigen (NS1) and serology (IgM).

- Blood, urine, and sputum cultures as appropriate.

- Leptospira antibody (IgM).

- Scrub typhus antibody (IgM)—Rapid immunochromatographic test.

- Blood culture-preferably a pair.

- Widal and typhi dot (if duration of fever $>7$ days).

- Liver and renal profile.

- USG to rule out liver abscess.

- Depending on local epidemiology, further specific investigations for appropriate organisms should be done.

\section{Step 2: Take Focused History}

- Tropical infections can have a variety of nonspecific presentations and generalized constitutional symptoms.

- Specific symptoms characteristic of some organisms should be carefully looked for.

- Fever:

- Many tropical infections have febrile episodes, which are nonspecific (Table 53.1). Rarely, fever pattern can be diagnostic, such as alternate-day fever in tertian malaria (vivax or falciparum) and saddle back biphasic fever (dengue). Biphasic fever with the first phase lasting 5-7 days is followed by a second febrile phase for 1-2 days.

- Anorexia and weight loss:

- History of severe weight loss is present in some tropical infections such as tuberculosis, visceral leishmaniasis, brucellosis, giardiasis, and schistosomiasis. 
Table 53.1 Tropical

infections presenting as fever

\author{
Malaria \\ Typhoid \\ Dengue fever \\ Leptospirosis \\ Chikungunya \\ Viral hepatitis A and E \\ Typhus \\ Tuberculosis \\ Brucellosis \\ Hepatic amebiasis \\ Visceral leishmaniasis \\ Parasitic hyperinfection (Strongyloides) \\ Relapsing fever \\ Viral hemorrhagic fever \\ Yersiniosis \\ Plague \\ Tularemia \\ Trypanosomiasis
}

- Diarrhea and vomiting:

- Acute watery diarrhea is a presenting feature of cholera, Giardia, rotavirus, Cryptosporidium, Isospora, and Bacillus cereus (toxin).

- Bloody diarrhea occurs in amebic dysentery, enteroinvasive and enterohemorrhagic Escherichia coli, Shigella, Salmonella, Yersinia, Clostridium perfringens, and Campylobacter.

- Chronic diarrhea (>2 weeks) is characteristic of giardiasis, amebiasis, ileocecal tuberculosis, strongyloidiasis, schistosomiasis, and Trichuris infestation.

- Abdominal pain:

- Acute abdomen with features of peritonitis may be present in typhoid perforation and ruptured amebic liver abscess.

- Other tropical infections presenting as acute abdomen are amebic liver abscess, splenic rupture (malaria, typhoid), biliary colic (Ascaris), intestinal obstruction or volvulus (Ascaris), acute salpingitis (Chlamydia), and severe gastroenteritis.

- Jaundice:

- Jaundice with fever may be a presenting feature of certain tropical infections such as viral hepatitis, leptospirosis, typhus, typhoid, yellow fever, brucellosis, amebic liver abscess, miliary tuberculosis, malaria (hemolysis-G6PD deficiency), ascending cholangitis (Ascaris), and hemolytic uremic syndrome (Shigella, E. coli).

- Cough and dyspnea:

- These may be prominent in infections such as extensive pulmonary tuberculosis, amebic lung abscess, acute respiratory distress syndrome (scrub typhus, leptospirosis, malaria during late phase), diffuse intra-alveolar hemorrhage (dengue, leptospirosis, hemorrhagic fever), pulmonary hydatid disease, paragonimiasis, and pneumonic plague. 
- Headache:

- Most febrile illnesses - especially malaria, typhoid, and dengue fever-are accompanied by headache.

- Sore throat:

- Severe sore throat with painful swallowing is characteristic of Corynebacterium diphtheriae infection.

- Hematuria, dysuria, and renal colic:

- Some tropical infections such as schistosomiasis, renal tuberculosis, and chlamydial urethritis can present with hematuria.

- History of skin rash (see Step 3).

- Travel history: A list of places visited in recent past in chronological order should be elicited. Endemic infections in different geographical areas should be considered in differential diagnosis.

- Occupational history: Exposure to contaminated water source may be a clue to leptospirosis.

- Seasonal variation: Many tropical infections have propensity to occur during the monsoon season.

- Animal exposure: pet dogs (ticks—rickettsial infection, ehrlichiosis etc.)

\section{Step 3: Perform Focused Physical Examination}

- General examination: Examine for anemia, lymphadenopathy, jaundice, and edema.

- Skin: Many tropical infections present with skin manifestations, and these should be searched meticulously:

- Maculopapular rash_dengue, typhus, measles, and rubella.

- Urticaria—-strongyloidiasis and schistosomiasis.

- Petechial rash — typhus, meningococcemia, and viral hemorrhagic fever.

- Vesicles-chicken pox, herpes simplex, and herpes zoster.

- Eschar-Scrub typhus (Fig. 53.1).

- Abdomen: Examine for hepatosplenomegaly and abdominal distension:

- Predominant hepatomegaly_viral hepatitis, amebic liver abscess, leptospirosis, yellow fever, and brucellosis.

- Predominant splenomegaly-malaria, typhoid, typhus, visceral leishmaniasis, and hydatid disease.

- Abdominal distension could be due to ascites (tuberculosis) or dilated bowel loops (Shigella dysentery).

- Cardiorespiratory system:

- Relative bradycardia is a feature of typhoid and typhus fever.

- Pleural effusion (tuberculosis).

- Appearance of new murmurs-especially regurgitant-and infective endocarditis.

- Central nervous system:

- Confusion and decreased conscious level: cerebral malaria, typhoid, dengue fever, leptospirosis, typhus, rabies, and viral hemorrhagic fever. 
Fig. 53.1 Typical eschar suggestive of scrub typhus infection
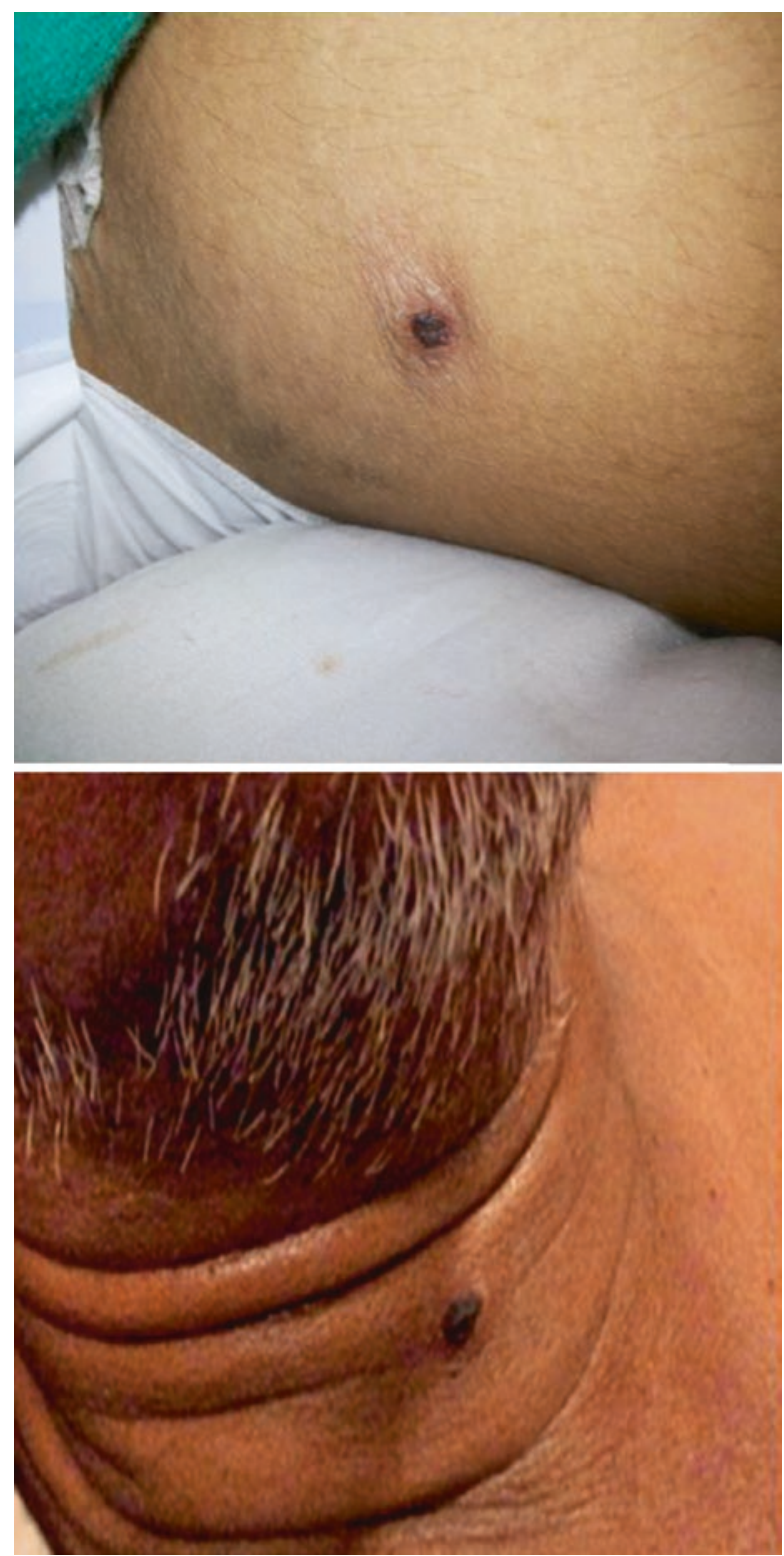

- Predominant encephalitic features: arbovirus, herpes simplex, measles, chickenpox, yellow fever, and rabies.

- Predominant meningitic feature: enterovirus, tuberculosis, amebiasis, and strongyloidiasis, bacterial.

- Seizures: cerebral malaria, schistosomiasis, neurocysticercosis, tuberculoma, and cerebral hydatid.

- Eyes: conjunctival infection, petechiae (leptospirosis and typhus), viral infection. 


\section{Step 4: Syndromic Approach to the Diagnosis and Management of Tropical Infections}

- Presenting syndrome

- Undifferentiated fever (up to 2 weeks): Malaria ( $P$. falciparum), scrub typhus, leptospirosis, typhoid, dengue and other common viral illness.

- Fever with rash/thrombocytopenia: Dengue, rickettsial infections, meningococcal infection, malaria (usually falciparum), leptospirosis, measles, rubella and other viral exanthem.

- Fever with ARDS: Scrub typhus, falciparum malaria, influenza including H1N1, leptospirosis, hantavirus infection, melioidosis, severe community acquired pneumonias due to Legionella spp. and Streptococcus pneumoniae and diffuse alveolar haemorrhage due to collagen vascular diseases.

- Febrile encephalopathy: Encephalitis (Herpes simplex virus encephalitis, Japanese B and other viral encephalitis), meningitis (S. pneumoniae, Neisseria meningitidis, Haemophilus influenzae, enteroviruses), scrub typhus, cerebral malaria and typhoid encephalopathy.

- Fever with multiorgan dysfunction: Bacterial sepsis, falciparum malaria, leptospirosis, scrub typhus, dengue, hepatitis A or E with fulminant hepatic failure and hepato-renal syndrome, Hanta virus infection, hemophagocytosis and macrophage activation syndrome.

- Send the investigation according to the presenting syndrome

- Fever with a rash/thrombocytopenia: dengue serology, platelet count, chikungunya serology, meningococcal serology, rickettsial serology, Widal test (typhoid), Epstein-Barr virus serology

- Fever with ARDS: Scrub typhus serology, malaria thick thin blood smears and antigen, H1N1 PCR testing, leptospirosis serology.

- Fever with multiorgan dysfunction: malaria parasite thick, thin blood smear and antigen, Leptospira serology, dengue serology, Legionella serology, varicella and influenza serology.

- Fever with encephalopathy-specific investigation: MP smear and antigen; lumber puncture; CT scan for tubercular meningitis, pyogenic meningitis, and viral encephalitis; herpesvirus serology and PCR.

- Specific tests

- Dengue

Viral isolation, viral RNA (RT-PCR) and Dengue NS1 (immunoassay) antigen becomes positive within first 4-5 days.

ELISA test for IgM antibodies (positive day 6) - IgG antibodies appear after 7-10 days and last for months to years. In the secondary dengue, IgG antibodies are present in high titer early in illness.

Detection of antibodies by hemagglutination inhibition assay: at least fourfold rise in titer of neutralizing antibodies in paired samples.

- Leptospirosis

Serology with the microscopic agglutination test is the gold standard with either a fourfold rise in titers between acute and convalescent serum or a single titer of more than 1:800 being diagnostic. 
Other serological tests are IgM antibody by an enzyme-based dot immunoassay with a sensitivity of $30 \%$ at 3 days and of $100 \%$ at 10 days into the illness.

Polymerase chain reaction (PCR) test for Leptospira antigen shows considerable promise.

Raised creatine phosphokinase levels is a supportive finding.

- Malaria

Three thick and thin smears 12-24 h apart should be obtained. The highest yield of peripheral parasites occurs during or soon after a fever spike; however, smears should not be delayed while awaiting fever spikes.

Thick smears are 20 times more sensitive than thin smears, but speciation may be more difficult. The parasitemia can be calculated based on the number of infected RBCs.

Thin smears are less sensitive than thick smears but facilitate speciation. This should be considered a qualitative test.

The quantitative buffy coat is a technique that is as sensitive as thick smears. Malarial antigen-immunochromatographic tests based on antibodies to malarial antigen like histidine-rich protein-2 (Pf HRP2), parasite LDH (pLDH), or plasmodium aldolase appear to be very sensitive and specific. RDT has sensitivity and specificity of $>95 \%$. Malaria is ruled out if two RDTs respectively are negative.

- Rickettsial infection

Look for eschar (black crust, 5-20 mm) - found in 40\% cases.

IgM Scrub Typhus

Weil Felix OXK: 1 in 320 or more (highly specific, poor sensitivity. Serology for typhus fever-Antibody for $65 \mathrm{KD}$ antigen. Rapid immunochromatographic test has sensitivity and specificity of $80-90 \%$.

Indirect fluorescent antibody: "Gold standard".

- Enteric fever

The diagnosis of typhoid fever is primarily clinical.

Cultures are widely considered $100 \%$ specific. Cultures of bone marrow is 90\% sensitive until at least 5 days after commencement of antibiotics. Blood, intestinal secretions and stool cultures are positive in $85-90 \%$ of patients who present within the first week of onset. Multiple blood cultures (>3) yield a sensitivity of 73-97\%. Large volume (10-30 ml) blood culture may increase the likelihood of detection.

- Special investigations

Procalcitonin, ESR, CRP.

Aspirates, scrapings, and pustular fluid may be obtained for Gram staining and culture. When a herpes simplex virus infection is suspected, a Tzanck test may be performed by unroofing a lesion and taking a scraping of the lesion base.

Biopsy samples from nonhealing or persistent purpuric lesions: Biopsy of inflammatory dermal nodules, ulcers, and muscles (tropical pyomyositis) should be done.

HIV serology.

Imaging: chest x-ray, echo, ultrasound of abdomen, and CT scan (when indicated). 


\section{Step 5: Start General Supportive Care and Specific Organ Support}

- Many tropical infections are self-limiting. Close monitoring and general organ support in the initial days or weeks of viremia or parasitemia will salvage many patients.

\section{Step 6: Initiate Empirical Therapy Based on Initial Presentation}

- Specific therapy is available only for a few tropical infections.

- Empirical antibiotics based on syndromic approach and the endemicity of a particular infection in the geographical region, should be started at the time of admission. De-escalation of antibiotics should be done once specific infection is identified.

- Ceftriaxone and doxycycline are empirical antibiotics of choice to cover typhoid fever, leptospirosis and scrub typhus, once malaria is excluded.

- In case of contraindication to doxycycline, azithromycin should be used.

\section{Step 7: Start Specific Treatment Once the Diagnosis Is Confirmed}

- Dengue

- A protocol for intravenous fluid therapy has been developed by the World Health Organization (WHO).

- An initial bolus of 5\% dextrose in normal saline or Ringer lactate $(20 \mathrm{~mL} / \mathrm{kg}$ of body weight) is infused over 15 min, followed by continuous infusion (10$20 \mathrm{~mL} / \mathrm{kg} / \mathrm{h}$, depending on the clinical response) until vital signs and urine output normalize.

- Crystalloids are equally effective as colloids in fluid resuscitation.

- Normalization of the hematocrit is an important goal of early fluid repletion.

- However, a normal or low hematocrit may be misleading in patients with overt bleeding and severe hypovolemia.

- Close clinical observation is essential, even after normal blood volume is restored, because patients can develop shock for 1-2 days after initial fluid resuscitation, which represents the period of increased vascular permeability in dengue hemorrhagic fever.

- Management of fever:

Control fever with paracetamol, cold sponging, and cold IV fluids.

Avoid aspirin and nonsteroidal anti-inflammatory drugs due to bleeding risk and risk of developing Reye syndrome (encephalopathy).

- Manage shock and multiorgan failure. 
- Manage secondary infections.

- Manage complications.

- Clinical guides for bleeding.

Bleeding time

Fundus examination - retinal hemorrhages

Hess capillary test/Tourniquet test: positive is more than 20 petechiae in an area of 1 in. when the blood pressure cuff is inflated midway between systolic and diastolic blood pressure for $5 \mathrm{~min}$.

- Platelet transfusions need to be given for symptomatic thrombocytopenia.

- Platelet transfusions have not been shown to be effective in preventing or controlling hemorrhage but may be warranted in patients with severe thrombocytopenia $\left(<10,000 / \mathrm{mm}^{3}\right)$ and active bleeding. Prophylactic platelet transfusions in patients with severe thrombocytopenia but without active bleeding are generally not recommended.

- Manage complications of fluid therapy in dengue fever.

- A decrease in hematocrit together with stable hemodynamic status and adequate urine output indicates hemodilution and/or reabsorption of extravasated fluids.

- Judicious use of intravenous fluids with proper monitoring is recommended.

- Fluid therapy may have to be discontinued if required, immediately, to avoid pulmonary edema, electrolyte imbalance, hypo- or hypernatremia, and hyperchloremic metabolic acidosis.

- Leptospirosis

- Treatment involves the use of crystalline penicillin at a dose of six million units daily or ceftriaxone $1 \mathrm{~g}$ every $12 \mathrm{~h}$.

- In penicillin-allergic patients, intravenous or oral doxycycline, $100 \mathrm{mg}$ every $12 \mathrm{~h}$, can be used.

- Manage shock, disseminated intravascular coagulation, and multiorgan failure.

- Scrub typhus

- Doxycycline is the drug of choice.

- In case of small children and pregnant women, azithromycin is the drug of choice.

- Rifampicin has also been used as an alternative drug.

- Enteric Fever

- Ceftriaxone i.v. 50-75 mg/kg/day for 10-14 days is the drug of choice to cover MDR S. typhi. Azithromycin and Ciprofloxacin are alternatives.

- Consider dexamethasone $3 \mathrm{mg} / \mathrm{kg}$ followed by $1 \mathrm{mg} / \mathrm{kg} 6$ hourly for $48 \mathrm{~h}$ in selected cases with encephalopathy, hypotension or DIC.

- Falciparum malaria

- For Plasmodium falciparum infections acquired in areas without chloroquineresistant strains, patients should be treated with oral chloroquine. A chloroquine dose of $600 \mathrm{mg}$ base (=1000 mg salt) should be given initially, followed by 
$300 \mathrm{mg}$ base (=500 mg salt) at 6,24 , and $48 \mathrm{~h}$ after the initial dose for a total chloroquine dose of $1500 \mathrm{mg}$ base (=2500 mg salt).

- For chloroquine-resistant strains, treatment options are as follows:

Artemisinin derivatives clear parasites very rapidly, are now a key component of malaria treatment worldwide, and have been shown to reduce mortality in severe malaria compared with parenteral quinine. Artemisinin-based combination therapies, including artesunate-mefloquine, artemetherlumefantrine, artesunate-amodiaquine, and dihydroartemisinin-piperaquine, are highly efficacious.

Under the CDC protocol, intravenous artesunate is administered in four equal doses of $2.4 \mathrm{mg} / \mathrm{kg}$ of body weight over a period of 3 days. The dosing schedule recommended by the WHO entails doses every $12 \mathrm{~h}$ on day 1 and then once daily.

Up to 7 days of therapy may occasionally be indicated in very ill patients.

- Quinine sulfate: Quinine has a rapid onset of action and, in combination with tetracycline, doxycycline, or clindamycin, has been shown to be a very efficacious treatment option for $P$. falciparum infections acquired in regions with chloroquine-resistant strains. 


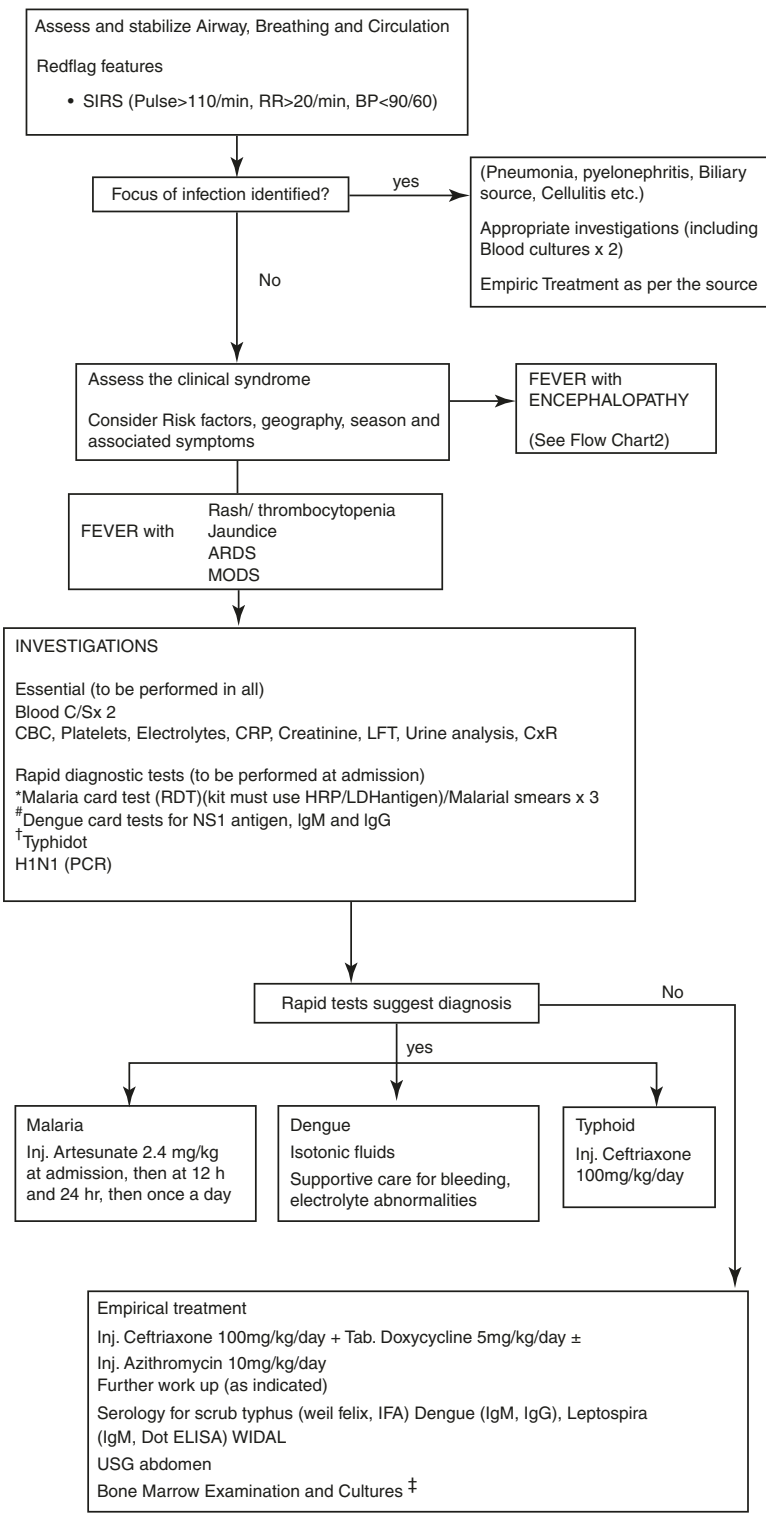

\footnotetext{
${ }^{*}$ Kit should use HRP/LDH antigens. Treat if RDT is positive. Malaria ruled out with if two negative RDTs.

${ }^{\text {NS }} 1$ antigen - day 1-5 of illness; IgG titre $>1: 1280$ is $90 \%$ sensitive and $98 \%$ specific.

$\dagger_{\text {Sensitivity } 95-97 \%}$

${ }^{\ddagger}$ Consider if fever already treated for $>1$ week or very high clinical suspicion of marrow involvement, hemophagocytosis
}

Flowchart 53.1 An algorithmic approach for the diagnosis and management of critical tropical infections. (Tropical fevers: management guidelines, IJCCM, 2014) 


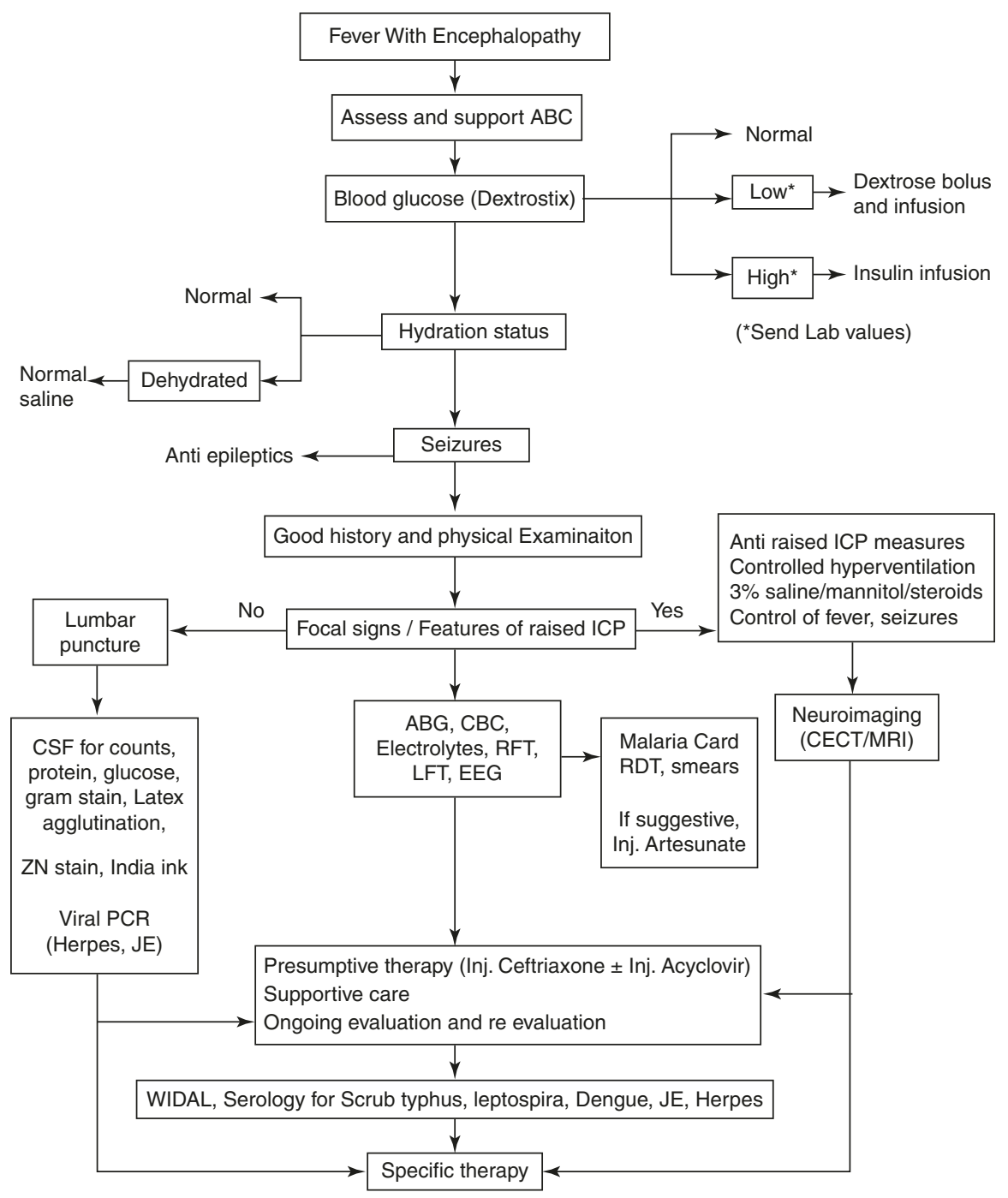

Flowchart 53.2 Algorithmic approach to fever with encephalopathy. (Tropical fever: management guidelines, IJCCM, 2014)

\section{Suggested Reading}

Banukumar S, Kannan I, Premavathi RK, Shantha S. Study on incidence of leptospirosis by serodiagnosis in rural population in the district of Kanchipuram, Tamilnadu, India. IJPCR. 2016;8(3):189-92.

Ganeshkumar P, Murhekar MV, Poornima V, et al. Dengue infection in India: a systematic review and meta-analysis. PLoS Negl Trop Dis. 2018;12(7) 
Karnad DR, Richards GA, Silva GS, Amin P, Council of the World Federation of Societies of Intensive and Critical Care Medicine. Tropical diseases in the ICU: a syndromic approach to diagnosis and treatment. J Crit Care. 2018;46:119-26.

Luce-Fedrow A, Lehman ML, Kelly DJ, et al. A review of scrub typhus (Orientia tsutsugamushi and related organisms): then, now, and tomorrow. Trop Med Infect Dis. 2018;3(1):8. A review article on Scrub Typhus.

Singhi S, Chaudhary D, Varghese GM, Bhalla A, Karthi N. Tropical fevers: management guidelines. Indian J Crit Care Med. 2014;18(2):62-9. Guidelines for the management of tropical fevers.

\section{Websites}

https://www.astmh.org/education-resources/tropical-medicine-q-a/major-tropical-diseases https://www.who.int/topics/tropical_diseases/en/ 